\title{
An Ultra Low Noise Telecom Wavelength Free Running Single Photon Detector Using Negative Feedback Avalanche Diode
}

\author{
Zhizhong Yan, ${ }^{1}$, a) Deny R. Hamel, ${ }^{1}$ Aimee K. Heinrichs, ${ }^{1}$ Xudong Jiang, ${ }^{2}$ \\ Mark A. Itzler, ${ }^{2}$ and Thomas Jennewein ${ }^{1, b)}$ \\ 1) Institute for Quantum Computing, University of Waterloo, \\ 200 University Avenue W, Waterloo N2L 3G1, Canada \\ 2) Princeton Lightwave Inc., 2555 US Route 130 S., Cranbury, \\ NJ 08540 USA
}

It is challenging to implement genuine free running single photon detectors for the $1550 \mathrm{~nm}$ wavelength range with simultaneously high detection efficiency (DE), low dark noise, and good time resolution. We report a novel read out system for the signals from a negative feedback avalanche diode (NFAD) ${ }^{1 / 3}$ which allows useful operation of these devices at a temperature of $193 \mathrm{~K}$ and results in very low dark counts $(\sim 100$ CPS $)$, good time jitter $(\sim 30 \mathrm{ps})$, and good DE $(\sim 10 \%)$. We characterized two NFADs with a time correlation method using photons generated from weak coherent pulses (WCP) and photon pairs produced by spontaneous parametric down conversion (SPDC). The inferred detector efficiencies for both types of photon sources agree with each other. The best noise equivalent power of the device is estimated to be $8.1 \times 10^{-18} \mathrm{~W} \cdot \mathrm{Hz}^{-1 / 2}$, more than 10 times better than typical $\mathrm{InP} /$ InGaAs SPADs show in free running mode. The afterpulsing probability was found to be less than $0.1 \%$ per ns at the optimized operating point. In addition, we studied the performance of an entanglement-based quantum key distribution (QKD) using these detectors and develop a model for the quantum bit error rate (QBER) that incorporates the afterpulsing coefficients. We verified experimentally that using these NFADs it is feasible to implement QKD over $400 \mathrm{~km}$ of telecom fibre. Our NFAD photon detector system is very simple, and is well suited for single-photon applications where ultra-low noise and free-running operation is required, and some afterpulsing can be tolerated.

\footnotetext{
${ }^{a)}$ Electronic mail: zyan@iqc.ca

b)Electronic mail: thomas.jennewein@uwaterloo.ca
} 


\section{INTRODUCTION}

Single photon detectors (SPD) are important devices in many research fields, such as optical quantum computing ${ }^{4}$, quantum cryptography ${ }^{\sqrt{56}}$, spectroscopy, fluorescence lifetime measurements ${ }^{7}$, and light detection and ranging $(\text { LIDAR })^{8}$. The telecom wavelength range around $1500 \mathrm{~nm}$ is of particular interest for various reasons. For example, quantum key distribution (QKD) via optical fiber must operate at the lowest photon transmission loss at $1550 \mathrm{~nm}$ in order to achieve the longest distance.

SPDs can be operated in two distinct modes: gated $^{9}$ or free running 10 14. Free running detectors are very important in certain applications, particularly where photons arrive in a random fashion, rather than synchronized on a regular clock time basis. For example, this is the case for photon pairs produced by spontaneous parametric down conversion (SPDC) with a continuous wave pump.

In the telecom wavelength range there exist several good candidates for free running SPDs, including photomultiplier tubes $(\mathrm{PMT})^{15}$, passively quenched InP/InGaAs SPADs ${ }^{16}$, shallow junction silicon SPADs using frequency upconversion ${ }^{17}$, transition edge sensors $(\text { TES })^{18}$, and superconducting nanowire single photon detectors (SNSPDs) $)^{19}$.

The SNSPDs currently have the best overall performance, such as low darkcount rates (DCR $<100 \mathrm{~Hz}$ ) and good timing jitter $(<65 \mathrm{ps})$. However, owing to their superconducting temperature requirements (under $4.2 \mathrm{~K}$ ), cryogenic systems are required. Moreover, the detection efficiency (DE) of SNSPDs is dependent on the polarization of the input photons ${ }^{20121}$. On the other hand, negative feedback avalanche diodes (NFADs), a recently developed type of InP/InGaAs APD (Princeton Lightwave Inc), can be operated in free running mode without cryogenic temperatures. These devices possess an integrated passive quenching resistor that minimize the amount of avalanche charges produced by a photon detection (another approach to self-quenching in InP-based single photon APDs, which employs epitaxial barriers as a negative feedback mechanism ${ }^{22123}$ ). Furthermore, these APD detectors have no DE dependency on the input photon polarization.

The darkcount rate and timing jitter of NFAD detectors arise from some intrinsic material properties and the device structure. Their darkcount rates are induced by the size of their avalanche region, as well as the trap-assisted tunneling effect ${ }^{2}$ (TAT). Timing jitter is generally dominated by the stochastic nature of the avalanche breakdown process. However, 
the external operation setup can be optimized to achieve a high signal-to-noise ratio (SNR) and good time resolution. We developed a novel readout system for the NFADs, which features (a) very low operating temperature; and (b) minimal avalanche charges required to trigger the readout circuit. This allows our system to achieve ultra low darkcount rates.

The significant features of our readout method are: first, the NFAD operates in a genuine free running mode; there is no active quenching circuitry involved to impose additional deadtime (which is usually needed to suppress the afterpulsing effect and to lower darkcount rates); Second, a pulse transformer is employed to isolate the NFAD bias circuitry from the high speed RF amplifiers to read out the photon electric signals. Instead of conventional capacitive coupling, we use inductive coupling, so that only the variation of the avalanche current is coupled to the external amplifier. This dramatically improves the sensitivity of the readout.

In this work two different NFAD devices are studied ${ }^{3}$. Detector I/NFAD 1 (model no. E2G6) has a feedback resistor of $1.1 \mathrm{M} \Omega$ and a diameter of $22 \mu \mathrm{m}$. Detector II/NFAD 2 (model no. E3G3) has the same nominal feedback resistor, but a larger device diameter of $32 \mu \mathrm{m}$.

The paper is organized as follows: we start with the experimental setup and device details. Then the "Simulation Program with Integrated Circuit Emphasis" (SPICE) model of our readout circuit including the NFAD equivalent circuit is simulated and compared with the experimental results. This is followed by a measurements of the DE, which is later verified using two NFADs to detect photon pairs generated by SPDC. Next the results from the afterpulsing, deadtime, and timing jitter measurements are presented. Lastly, we study the feasibility of using our NFADs to perform entangled photon QKD over distances of more than $400 \mathrm{~km}$.

\section{THE DETECTOR SYSTEM}

\section{A. Setup}

Fig. 11 depicts the detailed design of our in-house developed readout circuit. The entire system is comprised of two parts. The NFAD and its bias circuitry is inside a deep freezer (Global Cooling, ULT-25) with a lowest base temperature of $187 \mathrm{~K}$. At this base temperature, 


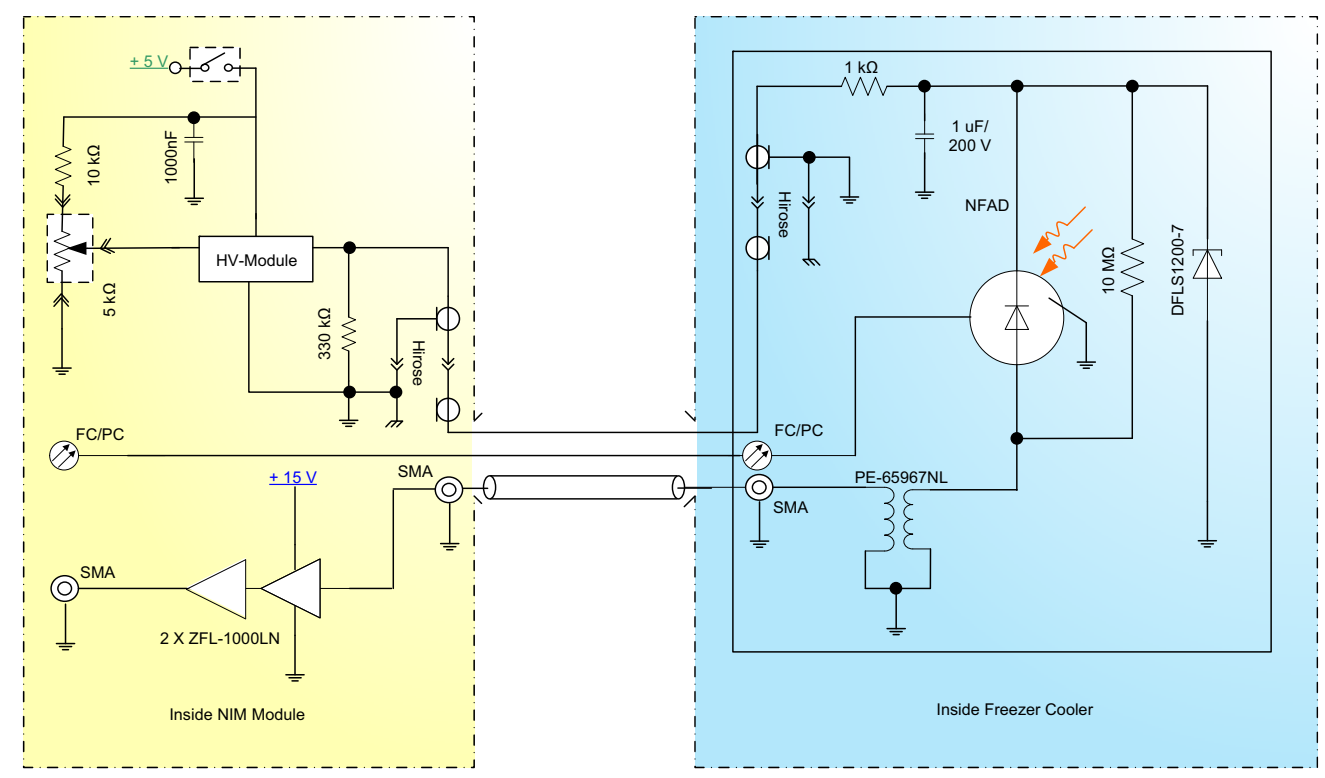

FIG. 1. The system design diagram of the entire NFAD readout circuits and connections. All the components in the right panel are inside a deep freezer. The ones in the left panel are at room temperature. SMA is the high frequency connector; FC/PC is the single mode fiber connector; Hirose is the NFAD high voltage cable connector.

the detector operating temperature is $193 \mathrm{~K}$. The primary coil of the pulse transformer (Pulse Electronics) is connected between the system ground and the cathode of the NFAD. The secondary coil of the transformer couples to a $50 \Omega$ transmission line (TL). A bypass resistor of $10 \mathrm{M} \Omega$ and a Schottky diode serve to protect the NFAD from unexpected voltage surges or spikes.

The rest of the readout electronics, including high voltage modules and the wide band RF amplifier, are in a nuclear instrumentation module (NIM) at room temperature. The high voltage control module (Matsusada) supplies variable bias voltage to the NFAD, and its output value is controlled by a multi-turn linear potentiometer. The bias voltage spans from 0 to $80 \mathrm{~V}$. Two wide band RF amplifiers (Mini-Circuits) have $40 \mathrm{~dB}$ gain in total to amplify the signals from the pulse transformer via the TL.

The experimental setup to characterize the NFADs is based on time-correlated single photon counting (TCSPC), see Fig. 2. Optical pulses are generated with an id-301 $1550 \mathrm{~nm}$ distributed feedback (DFB) laser, attenuated by an EXFO programable attenuator (FVA3100). The laser is triggered by a two-channel function generator (Tektronix AFG3502). The attenuated weak coherent pulses pass through the attenuator and are sent to the NFAD. 


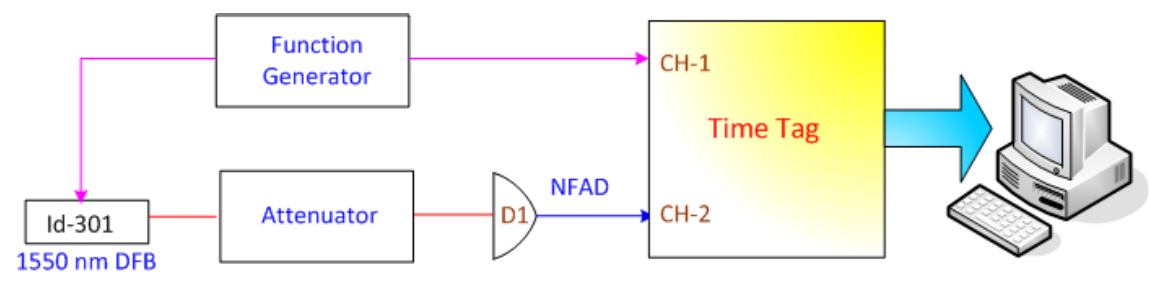

FIG. 2. Testing setup for characterization of the NFAD using the time-correlated single photon counting method. Details in the text.

The time tag unit records detections and the synchronization signals, and establishes their coincidences.

\section{B. The photon detection pulse responses}

The photon detection response is measured by a fast digital oscilloscope. Fig. 3 (a) shows a typical waveform from an experimental measurement of the single photon response. We also simulated the response using a SPICE model for the equivalent circuit depicted in Fig. 3 (b) and the NFAD model based on the results of Hayat et al. 24 . The simulation allows us to estimate the heating induced by each photon detection event, as well as the amount of avalanche charges coupled to the external readout circuit. Both estimations can be achieved by simulating transient response simulation of a single avalanche event.

The simulation results for the signal response of two readout methods are illustrated in Fig. 3. (c) shows the transient response based on our readout circuit design (see Fig. 3 (b)); graph (d) is the result for the conventional readout method shown in Ref. 1. The conventional circuits transient response has a persistent current plateau ranging from $\sim 10$ $100 \mathrm{~ns}$ and is proportional to the overbias voltage ${ }^{25}$. In comparison, our main response voltage peak is less than $5 \mathrm{~ns}$, and is independent of overbias voltage. The slow variations of the persistent current induce almost no voltage via the transformer to the amplifier input. The simulation and experiment results agree well for the leading period of about $5 \mathrm{~ns}$ long. The experimental data exhibit a more complicated response, because the current SPICE model only accounts for the main physical process.

In order to study the power consumption arising from the Joule heating effect, we performed numerical integration over the product of total power supply current and voltage. Two sources of heat generation are identified: a constant background heat power 


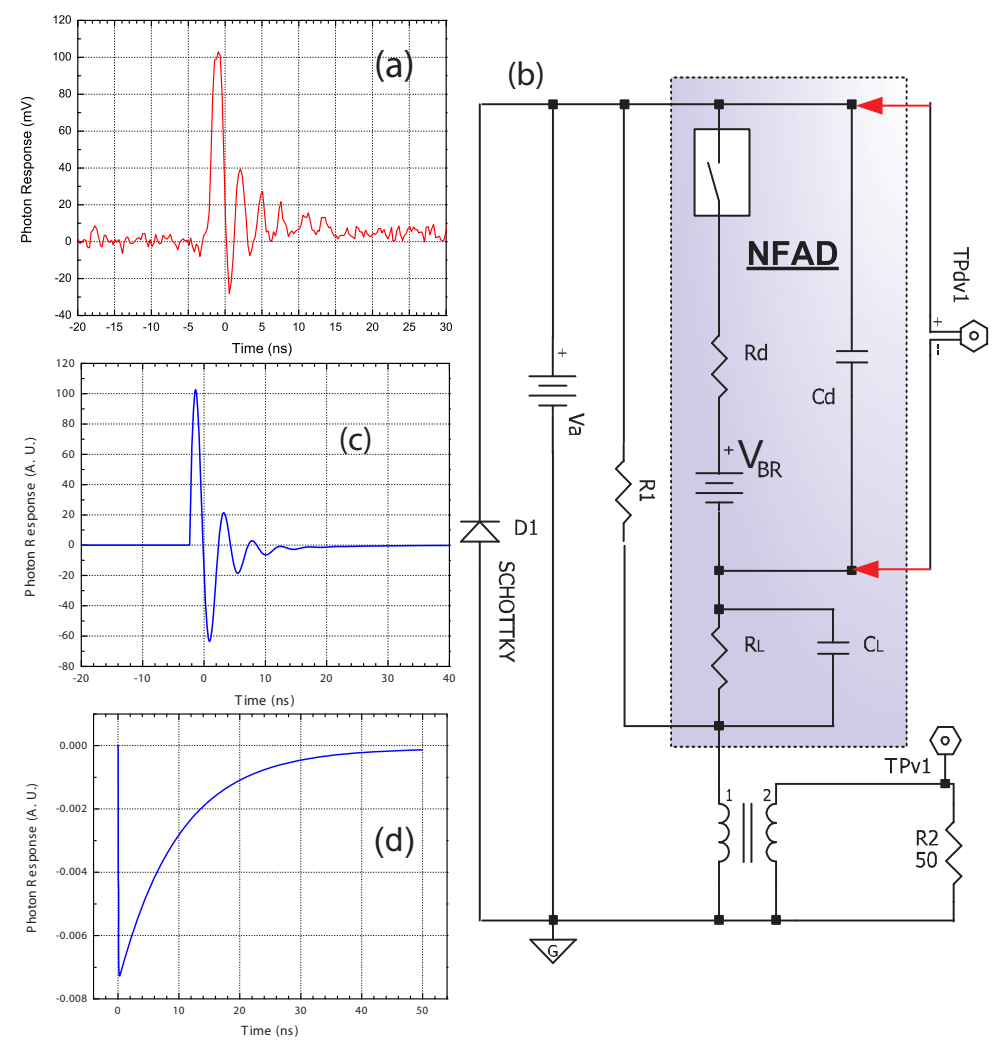

FIG. 3. (a) The experimental single photon response recorded with a fast digital oscilloscope after the $40 \mathrm{~dB}$ gain amplification. (b) The SPICE model for the NFAD read out circuit. The dash line square highlights the NFAD equivalent circuit components; The testing points TPv1 and TPdv1 represent photon detection response output and intrinsic SPAD voltage drop, respectively; the voltage source VBR represents the breakdown voltage of the NFAD. (c)-(d) are results of the transient simulation of a single photon responses, before the amplifier. (c) is the simulated response for the readout circuits used in this work; (d) is the simulated response with the conventional readout methods using a bias-tee in Ref. 1, The model parameters for the bias tee in the the conventional method are simplified from the ones provided by its manufacturer.

of $\sim 5 \times 10^{-4} \mathrm{~W}$ induced by bias and protection circuitry (e.g. $10 \mathrm{M} \Omega$ resistor $\mathrm{R}_{1}$ ), and an additional $4 \times 10^{-12} \mathrm{~J}$ produced by every detection. Therefore, even at very high detection rates, the heating caused by detections is negligible, compared to other sources of heat, such as the background heat.

Moreover, it is of interest to analyze the net avalanche charge, $Q_{\mathrm{av}}$, coupled to the external amplifier. We performed the numerical integration over the simulated voltage across a $50 \Omega$ load via $Q_{\mathrm{av}}=\frac{1}{50} \int_{0}^{\tau} \mathrm{V}_{\mathrm{TPv} 1} \cdot \mathrm{dt}$. The computed result showed the avalanche charge with 
inductive coupling is about $5 \times 10^{-16} \mathrm{C}$ (corresponding to $3 \times 10^{3}$ electron charge $e^{-}$). Comparing this to the net avalanche charge for a conventional readout circuit ${ }^{2}$, ours consumes at least two orders of magnitude less avalanche charges to produce a legitimate photon response pulse. This low coupling charge leads to the improved sensitivity of our readout circuit, and contributes to the low afterpulsing dark noise performance of the detector system.

\section{DETECTION EFFICIENCY CHARACTERIZATION}

\section{A. Repetitive weak coherent states method}

We first use attenuated coherent states, prepared at regular repetition rate, to characterize the detection efficiency of the NFAD. We choose the period of the repetitive pulse $\left(T_{0}=\right.$ $100 \mu \mathrm{s})$ to be much longer than the detector recovery time $(\tau=50 \mathrm{~ns})$. The coherent state

of each pulse is represented in the Fock basis by $|\alpha\rangle=e^{-\frac{|\alpha|^{2}}{2}} \sum_{n=0}^{\infty} \frac{\alpha^{n}}{\sqrt{n !}}|n\rangle$, where the average photon number contained in each pulse is $\mu=|\alpha|^{2}$. Since detection events are identified by comparing the voltage level of an photon response pulse to a pre-setting threshold, we model this type of detector as a so-called bucket detector ${ }^{26}$. The probability for click and noclick of the bucket detector are described by $\hat{f}_{\text {click }}=\sum_{n=0}^{\infty} 1-\left(1-\eta_{D}\right)^{n}|n\rangle\langle n|$ and $\hat{f}_{\text {no click }}=$ $\sum_{n=0}^{\infty}\left(1-\eta_{D}\right)^{n}|n\rangle\langle n|$ respectively.

In the experiment, we measure the ratio of detected photon click events, $R_{\text {det }}$, within the $1 \mathrm{~ns}$ correlated time window, $\tau_{0}$, versus the photon trigger repetition rate, $f_{\text {rep }}$, under a certain average photon number per pulse, $\mu$. We combine the coherent quantum states to derive the detection efficiency, $\eta_{\mathrm{D}}$ with the TCSPC method as:

$$
\eta_{\mathrm{D}}=-\frac{1}{\mu} \ln \left(\frac{1-\frac{R_{\mathrm{det}}}{f_{\mathrm{rep}}}}{1-d_{\mathrm{B}} \tau_{0}}\right)
$$

where the total non-photon detection events, $d_{\mathrm{B}}$, becomes a summation of pure dark counts, $D$, and afterpulsing events induced by photon detections, $d_{\mathrm{afp}}$, i.e. $d_{\mathrm{B}}=D+d_{\mathrm{afp}}$. $\tau_{0}$ is the smallest time window in the TCSPC counting system. Here we always use a 1 ns time window for all measurements with the time tag unit.

Because the darkcount and repetition rates are both below $10 \mathrm{kHz}$, and $\tau_{0}=10^{-9} \mathrm{~s}$, we are able to use $1-d_{\mathrm{B}} \tau_{0} \simeq 1$ as a very good approximation in most of our DE measurements. 
Thus, we have a practical relation to compute the DE:

$$
\eta_{\mathrm{D}} \simeq-\frac{1}{\mu} \ln \left(1-\frac{R_{\mathrm{det}}}{f_{\mathrm{rep}}}\right) .
$$

Here we assume the detector operator is known to behave as a bucket detector. If one wants to characterize a completely unknown detector, then a more general approach would be to perform the detector tomography 2728 .

Prior to the detection efficiency (DE) measurements, we first measure the darkcount rate for various bias voltages. Both detectors exhibit a similar behavior of voltage to darkcount rate. Fig. 4 shows the trend of darkcount rate as a function of input voltage. Over the range of overbias voltages tested, the darkcount rate varies from 0.1 to 10,000 CPS.

The DE testing conditions are as follows: the laser source, id-301, is triggered at $1 \mathrm{kHz}$ with its output attenuated such that the photon flux is determined to be 1 photon per pulse by a calibrated power meter ${ }^{29}$. We then verify this attenuation using a commercial detector $^{30}$, id-201, set to $10 \%$ detection probability with a 20 ns gate and a 40 ns deadtime. We verify that the ratio of detected photon counts vs. total triggering count reaches $9.5 \%$. At this ratio at which, based on coherent state detection probability calculation, the average photon number is $\mu=1$. The power meter and the id-201 were both employed for the initial calibration only, and these two independent approaches agree each other within the error bars in the DE measurement results. In subsequent measurements, we use the attenuator only to set the photon flux.

The photon event, detected by the NFAD, is registered by a time tag unit whose time resolution is $156 \mathrm{ps}$. The time window width was set to be $1 \mathrm{~ns}$ for all of our measurements, unless explicitly mentioned. Each measurement was carried out for a second and repeated 10 times. Fig. 5 shows the DE measurement results using Eq. 2. The experimental DE exhibits a saturation at darkcount rate above $10 \mathrm{kHz}$. The maximum detection efficiency for the NFADs is $\sim 14 \%$, and, with reasonable darkcount rates, it can reach $\sim 10 \%$.

The saturation of detection efficiency (at about $14 \%$, illustrated in Fig. 5) is actually due to the run-away increase in apparent DCR by afterpulsing. However, there is no way to separate the random darkcount events from the afterpulses that they induce. The afterpulsing process is highly non-linear, since afterpulse events can induce additional afterpulsing events. Hence, there is always a point at which afterpulsing appears to increase quite dramatically with any additional bias. Nonetheless, it is expected that NFADs can provide significantly 

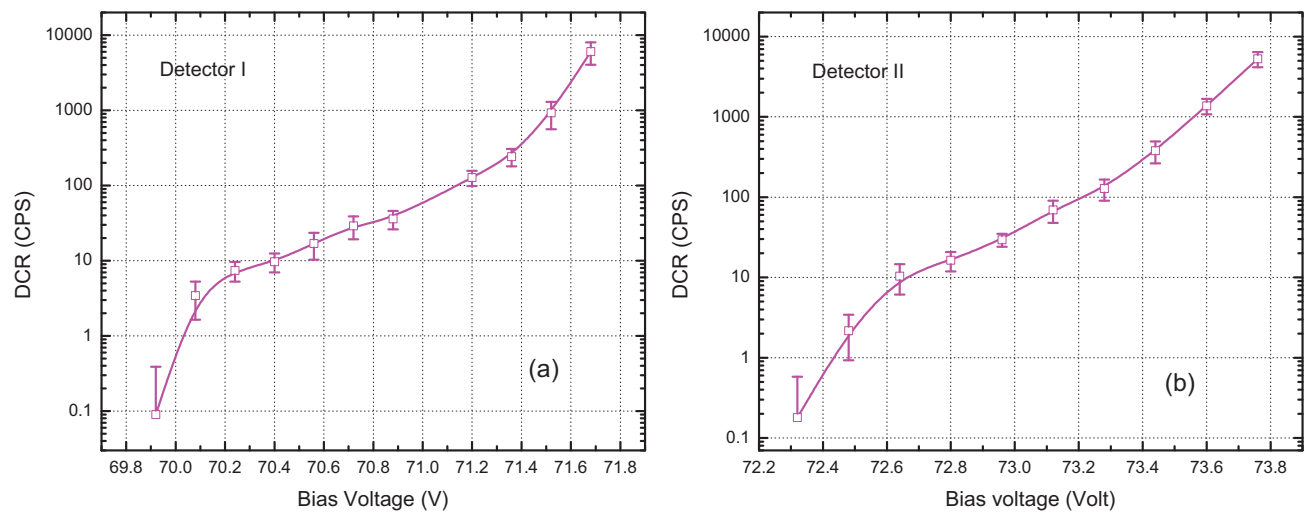

FIG. 4. Measurement of the darkcount rates for the two NFAD devices. Both are measured at 193 $\mathrm{K}$ with the same discriminator voltage settings.
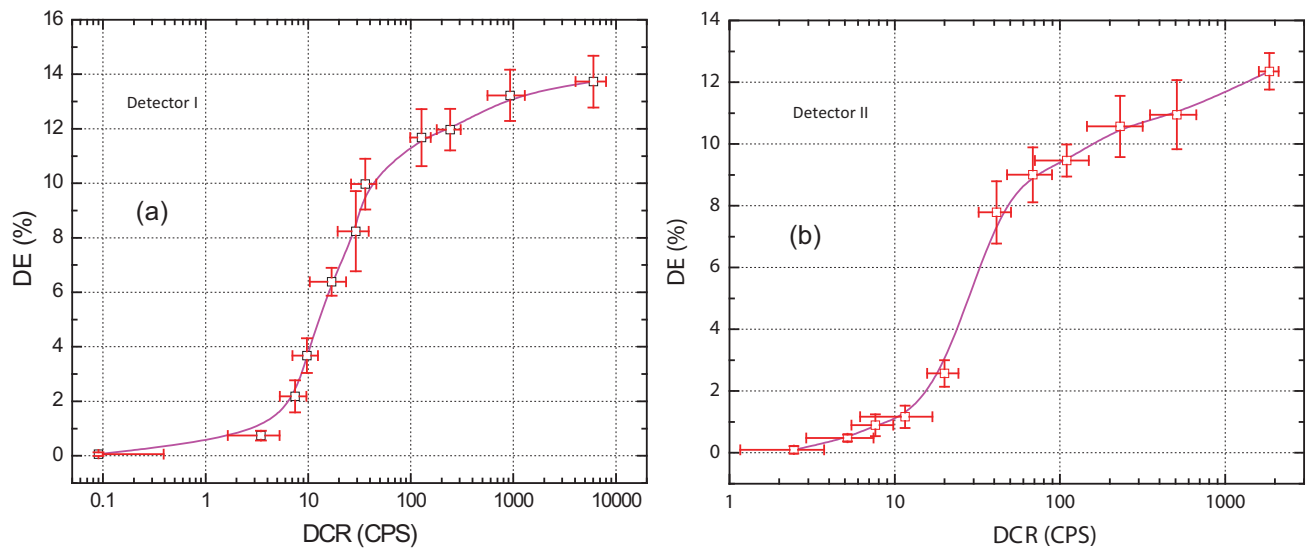

FIG. 5. DE vs Dark count measurements results for two NFAD detectors. The discriminator threshold voltage level is set to $100 \mathrm{mV}$, and the testing temperature is $193 \mathrm{~K}$.

higher DE values (> 25\%), similar to that of the id-201 detector, when operated in the gated mode with a sufficient hold-off time, although this imposes a reduction in detector "availability", that is undesirable for some experiments.

Note that this detector efficiency is at the system level; no normalization was used to take into account the device active area, nor the insertion loss of the coupling path. In the DE test for both NFADs, we also vary the discriminator voltage levels. No significant DE improvements can be found by choosing different threshold values.

We verify that the detectors act as bucket detectors. If this hypothesis is correct, then the detector click probability (the ratio of the detection event counts within the correlation time window to that of the total pulsed photon rate) is expected to behave as $f(\mu)=$ 

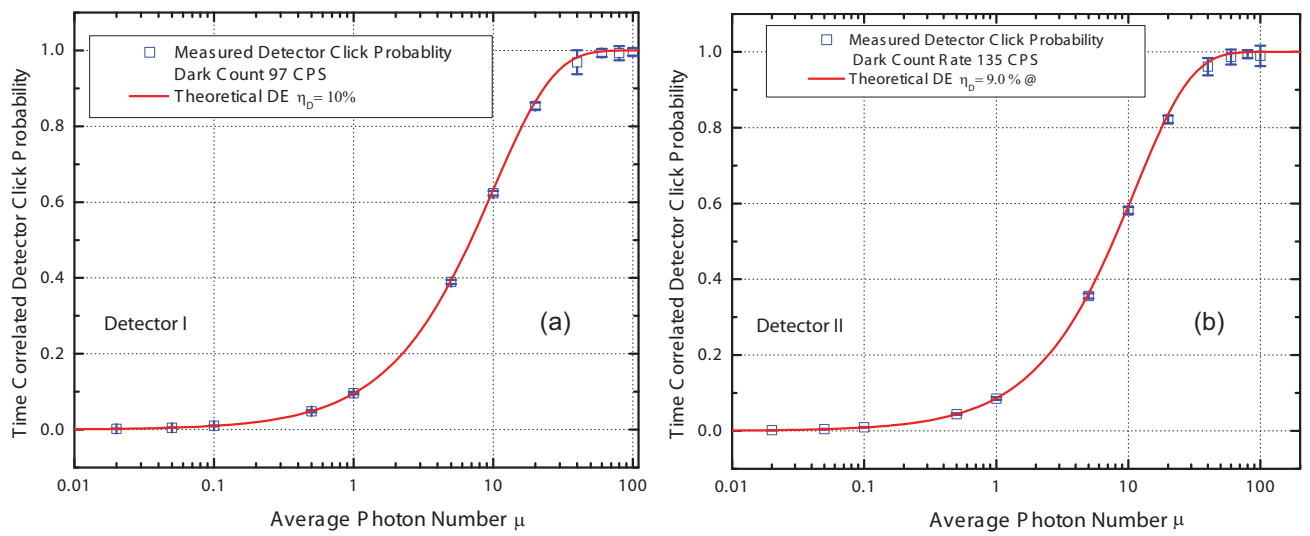

FIG. 6. Verification of bucket detector model. The hollow squares are the experimental results. The solid lines are the simulation results based on modeling the detector as a bucket detector.
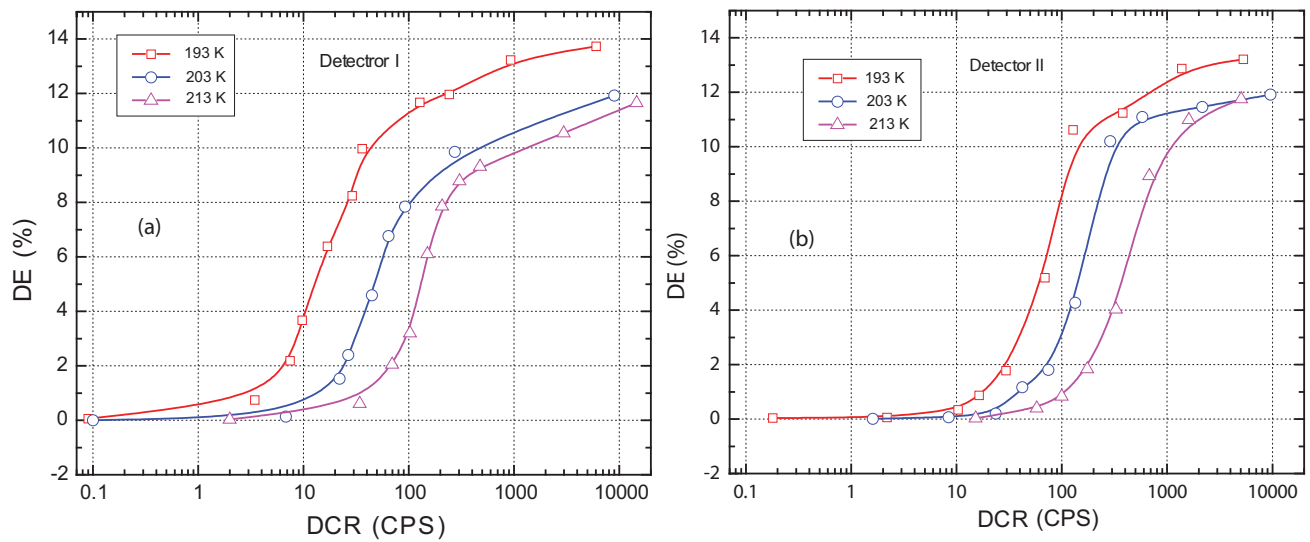

FIG. 7. Effect of temperature on detection efficiency and dark counts. The data points represent experimental measurements, and the lines are simple smooth fits.

$1-\exp \left(-\eta_{\mathrm{D}} \cdot \mu\right)$. The experimental results are compared with theoretical curve in Fig. 6. The curve is computed with the best fitting value of $\mathrm{DE}, \eta_{\mathrm{D}}$. The measurement results for two different NFADs are obtained at the darkcount rate approximately at 100 CPS. The other testing conditions are kept the same the DE measurement, except for the photon triggering rate, which is increased to $10 \mathrm{kHz}$. The good agreement for both detectors indicate that the bucket detector model is valid for our NFADs. This description is thus appropriate for modeling further quantum optics measurements.

A key benefit of our unique readout circuit is that we operate the NFAD at lower temperature than most InGaAs SPADs are operated at (typically around $233 \mathrm{~K}$ ). Fig. 7 shows the effect of temperature on the relation between DE and DCR. Once the DE and its associated 
darkcount rate are measured, we can find the detector noise equivalent power (NEP) via $\mathrm{NEP}=\frac{h \nu}{\eta_{\mathrm{D}}} \sqrt{2 D}$. The best noise equivalent power is estimated to be $8.1 \times 10^{-18} \mathrm{~W} \mathrm{~Hz}^{-1 / 2}$ for Detector 1 ; and $1.4 \times 10^{-17} \mathrm{~W} \mathrm{~Hz}^{-1 / 2}$ for Detector 2 at $193 \mathrm{~K}$. We will also study the figure of merit, which depends on the detector jitter time, in Section V.

\section{B. DE measurements using a continuous wave pumped SPDC source}

Many quantum communication protocols are based on entangled photon pairs created from SPDC. It is of interest to characterize NFADs using photons from a SPDC source for several reasons: the non-Poissonian photon distribution of SPDC source behaves with a thermal-like property $\stackrel{31}{3}$; Most importantly, correlated photons allow to verify the detection efficiency with least assumption on photon flux calibration ${ }^{32}$, and avoid such calibration uncertainties in the WCP scheme; Moreover, the photon pairs are created at random times, allowing us to convincingly demonstrate the free-running mode of NFADs.

The unique property of SPDC photon pairs has been exploited to characterize single photon detectors ever since the seminal work by Burnham et al. ${ }^{[33}$, other researchers have

published their approaches $32 \sqrt[34 \mid 35]{3}$ to reduce the uncertainties in determining the absolute detector efficiencies. The experimental accuracy is limited by a wide varieties of nonideal factors 32 . The typical experimental configuration for characterizing a SPDC source operated with a continuous-wave (CW) laser, uses two free running single photon detectors, and time correlation photon counting logics. Note that the production times of photon pairs are random in this case.

An alternative configuration ${ }^{36}$, which is widely used particularly when the down converted photon pairs in the $1550 \mathrm{~nm}$ wavelength range, can be the use of of one free running detector, in combination with a gated SPAD, which typically has a higher efficiency.

In our experiment, we tested two combinations of detectors, as displayed in Fig. 8. The SPDC source is an integrated waveguide based on a periodically poled lithium niobate (PPLN) substrate 37 . Fig. 8 (a) illustrates the first setup, which is comprised of two free running NFADs, with their detection events recorded by a time tag unit. Both free-running NFADs have the figure of merit $H>1 \times 10^{7}$ at $\lambda=1550 \mathrm{~nm}$, as defined in Ref. 38.

Fig. 8 (b) shows the commonly used configuration: a free running NFAD is employed to trigger the second gated id-201 ULN SPD module. Its settings are: detection efficiency is 


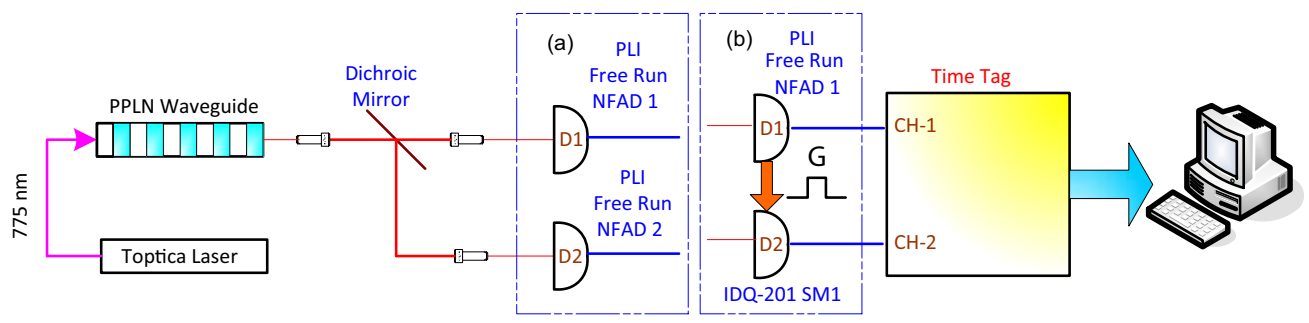

FIG. 8. Experimental setups used for two testing the NFAD 1 using SPDC. In dashed box (a), two free running NFAD detectors. In dashed box (b), one NFAD is placed in one of photon arms, a gated commercial detector (IDQ) is at the other arm. Photon detections from the NFAD are used to trigger an id-Quantique commercial single photon detector.
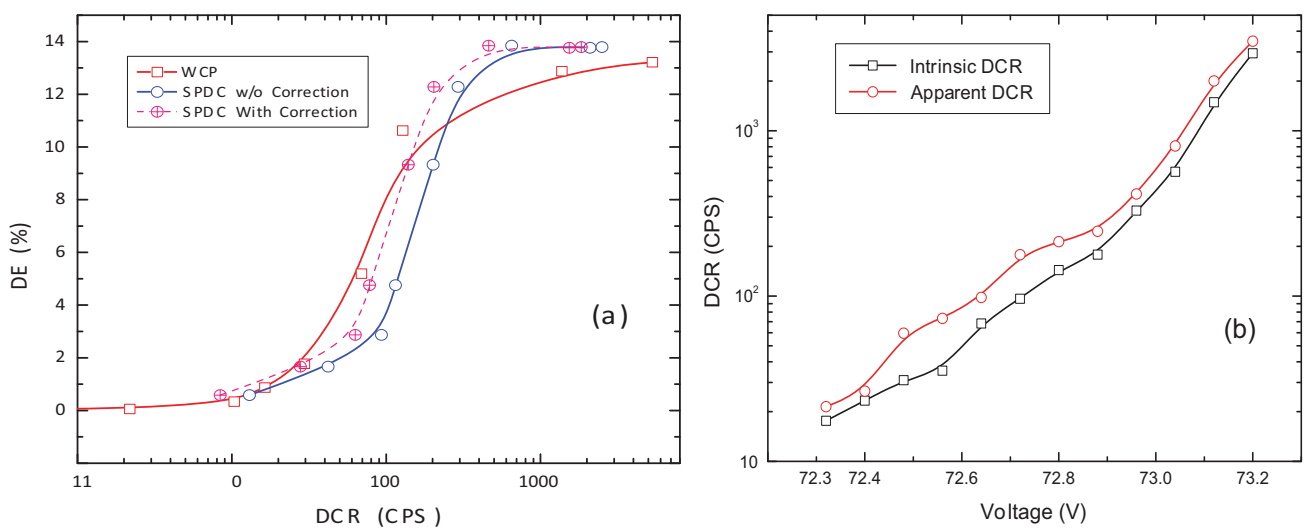

FIG. 9. (a) The inferred detection efficiency of the NFAD, from a direct comparison with an id-201 commercial detector. The solid line (hollow circle) is the inferred DE using the SPDC scheme at $1530 \mathrm{~nm}$; the dash line (cross circle) is the inferred DE corrected for stray light detection; the solid line (square) is the DE characterized by the WCP scheme at $1550 \mathrm{~nm}$. (b) The darkcount observed in the presence ( "apparent") and absence ("intrinsic") of stray light coupled into the optical fiber connecting to the detector when varying the bias voltage.

$10 \%$, deadtime is $40 \mu \mathrm{s}$, and gate width is $2.5 \mathrm{~ns}$. The SPDC in PPLN is set to be in the non-degenerated mode, where the two down converted photons have different wavelength of $(1570 \pm 12.5) \mathrm{nm}$ and $(1530 \pm 12.5) \mathrm{nm}$, respectively. The photons are separated by a dichroic mirror and fed into two detectors connected to channels 1 and 2.

The overall procedure is based on two successive measurements depicted in Fig. 8 (a) and (b). For the two free running detector scheme, we keep NFAD 1 at $6.1 \times 10^{3}$ counts per second on average. To achieve this condition, we maintain a constant bias voltage to 
NFAD 1 and a constant pumping power to the PPLN. We vary the bias voltage to the second detector NFAD 2. At each voltage step, we record the total single counts, as well as coincidental counts using the time tag unit.

The net count (total single rate minus darkcount rate) of the down converted photons in channel one (CH-1) and channel two (CH-2) are given by $N_{1}=\eta_{1} \eta_{D 1} N\left(1+\kappa_{1}\right), N_{2}=$ $\eta_{2} \eta_{D 2} N\left(1+\kappa_{2}\right)$; their coincidental counts $N_{c}$ is given by $N_{c}=\eta_{1} \eta_{D 1} \eta_{2} \eta_{D 2} N$, where $N$ is number of photon pairs, $\eta_{1}$ and $\eta_{2}$ are the efficiencies of the two channels, and $\eta_{D 1}$ and $\eta_{D 2}$ are the detection efficiencies of the two detectors; $\kappa_{1}$ and $\kappa_{2}$ are the afterpulsing coefficients of NFAD 1 and NFAD 2, which will be addressed in the following section. Therefore, from the scheme in Fig. 8 (a), we can find the ratio $\chi_{1}=\frac{\eta_{2} \eta_{D 2}^{\mathrm{NFD}}}{1+\kappa_{1}}=N_{c} / N_{1}$ using two NFADs; Subsequently, if we switch to the scheme in Fig. 8 (b), we can find another ratio $\chi_{2}=$

$\frac{\eta_{2} \eta_{D 2}^{\mathrm{IDQ}}}{1+\kappa_{1}}=N_{c}^{\prime} / N_{1}^{\prime}$. Therefore, we can infer the efficiency of NFAD 2 via $\eta_{D 2}^{\mathrm{NFAD}}=\eta_{D 2}^{\mathrm{IDQ}} \chi_{1} / \chi_{2}$. In practice, the afterpulsing coefficient is hard to maintain constant when the darkcount rate is high. Additionally, the accuracy of this detector efficiency measurement is also limited by wide varieties of other nonideal factors ${ }^{32}$.

Fig. 9 (a) displays the inferred detection efficiencies $\eta_{D 2}^{\mathrm{NFAD}}$ of NFAD 2, and the results are compared with the aforementioned WCP method. The two plots are at the same bias voltage and discriminator threshold settings. The calculation of $\eta_{D 2}^{\mathrm{NFAD}}$ is based on the assumption that the channel 2 efficiency $\eta_{2}$, and NFAD 1 afterpulsing coefficient $\kappa_{1}$, are both kept constant in two consecutive schemes. The DE inferred from the SPDC method is plotted against apparent darkcounts when the stray light photons are included. Those stray light photons impose no effect on the inferred DE values, but reduce the SNR of the NFADs. We performed a separate darkcount measurement to estimate the number of darkcounts caused by stray light shown in Fig. 9 (b). This allows us to correct the inferred DE values based on the substraction of stray light detection. The corrected DE (the dash line in Fig. 9 (a)) exhibits improved SNR, and the overall shape of the WCP and SPDC methods agrees.

\section{AFTERPULSING}

Afterpulsing is the most significant side effect for InGaAs SPADs; it introduces extra counts, in addition to the intrinsic photon and noise counts of the detector. It is mostly owing to defects in the semiconductor materials form carrier traps which hold the avalanche 
charge carriers. The lifetime of the trapped charge carriers ranges from a few ns up to some tens of $\mu$ s in conventional InGaAs/InP SPADs working in the gated Geiger mode ${ }^{39}$. As the operating temperature is lowered, such as the case in this work, the afterpulsing problem becomes more prominent because the trapping is longer.

The NFADs afterpulsing measurements are performed with the same setup as for the DE characterization (see Fig. 2). First, we measure the photon response using a fast digital oscilloscope instead of the time tagger. The measured screen shot is displayed in Fig. 10 (a). We also simulate the voltage across the intrinsic SPAD inside the NFAD with the SPICE circuit model (refer to Fig. 3 (b)). The transient simulation results is shown in Fig. 10 (b), where the time constant is determined to be $1.1 \mu \mathrm{s}(R=1.1 \mathrm{M} \Omega, C=1 \mathrm{pF})$.

We use the time tagger to measure the detector recovery. To identify which event is the photon detection, we measure a synchronization signal from the function generator on $\mathrm{CH}-1$ (see Fig. 22) as the time reference. This enables us to discriminate between photon detections and events caused by dark counts or afterpulsing. All of the afterpulsing measurements are taken with the NFAD darkcount rate ${ }^{40}$ set to about 100 CPS . Because we only record time tags for detector click event where the amplitude is higher than a preset threshold voltage, the weak avalanche events for the first few tens of ns after a detection are not recorded.

Fig. 10 (c) illustrates this effect as an effective detector deadtime, following the rising voltage of NFAD recovery process right after a photon detection event. Depending on the operating temperature and the discriminator voltage settings, we observed that the NFAD effective deadtime is at least 40 to $50 \mathrm{~ns}$ long (dash line 1). After the deadtime period, we find a rapid recovery of afterpulsing events, rising within a time of about 25 ns (between dash line 1 and line 2).

In Fig. 10 (d), we quantitatively measure the lifetime of the trapped avalanche carriers using a standard exponential decay function ${ }^{41}, C_{0} \exp \left[-\left(t-T_{\text {dead }}\right) / \tau_{\mathrm{d}}\right] . T_{\text {dead }}$, in our case, is the NFAD effective deadtime resulting from the finite threshold of the time tag unit. Theoretically, this value is around zero. With higher temperatures, the effective deadtime is slightly shorter, as shown in Fig. 10, $\tau_{\mathrm{d}}$ is the characteristic detrapping time. Our best fit value to this curve is $\tau_{\mathrm{d}}=1.1 \mu \mathrm{s}$ at both $193 \mathrm{~K}$ and $203 \mathrm{~K}$, while the prefactor $C_{0}$ is smaller at $203 \mathrm{~K}$. If we compare $\tau_{\mathrm{d}}$ with voltage recovery time constant, it turns out that they are almost identical, which implies that detrapping of the residual avalanche charge is mainly induced by the detector recovery voltage. 

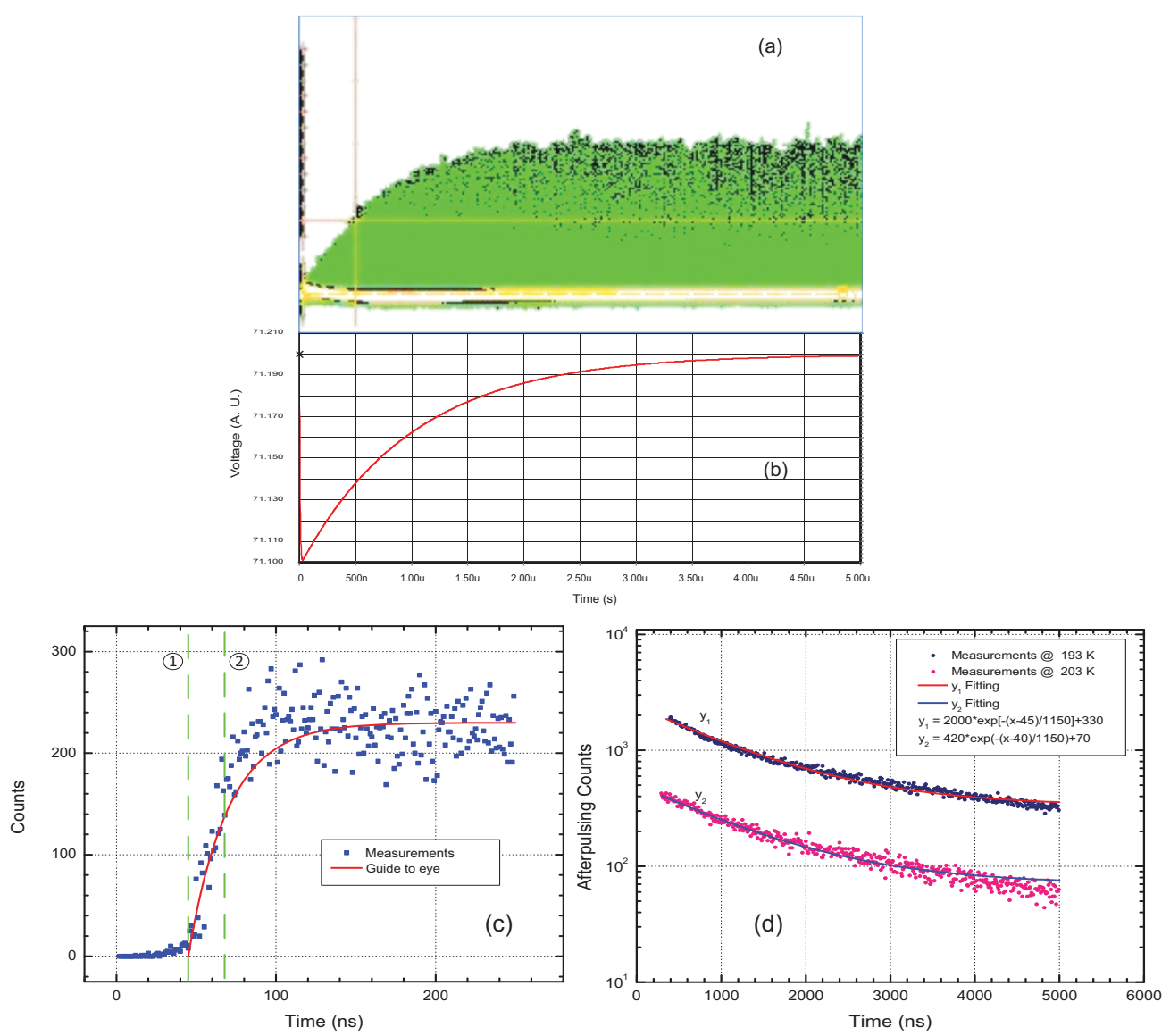

FIG. 10. (a)Accumulated measurements of the transient detector response; this plot is the screen capture from a fast oscilloscope collected for 6 hours; the saturated amplitude is about $300 \mathrm{mV}$. (b) The simulation for the transient voltage drop across an intrinsic SPAD inside the NFAD. Both plots share the same time scale of $5 \mu \mathrm{s}$. (c) Measurement of the detector deadtime and recovery time. The points are the measured photon detections in a 1 ns time resolution, and the solid line is the guide to eye. (d) Afterpulsing decay measurement and fitting. The random release of the trapped charges induced by photon detection events results in the afterpulsing probability decaying exponentially. The simulation curve is the solid line and the scattered dots are the accumulated measurement results for two temperatures.

Fig. 11 (a) shows the afterpulsing behavior of our NFADs for different discriminator voltages. We notice that increasing the threshold voltage can reduce the amount of afterpulsing for a short time after the detection, but the effect on the overall number of afterpulsing events is small. Fig. 11 (b) illustrates the effect of operating temperature on afterpulsing probability. As expected, afterpulsing becomes more prominent at lower temperatures. 

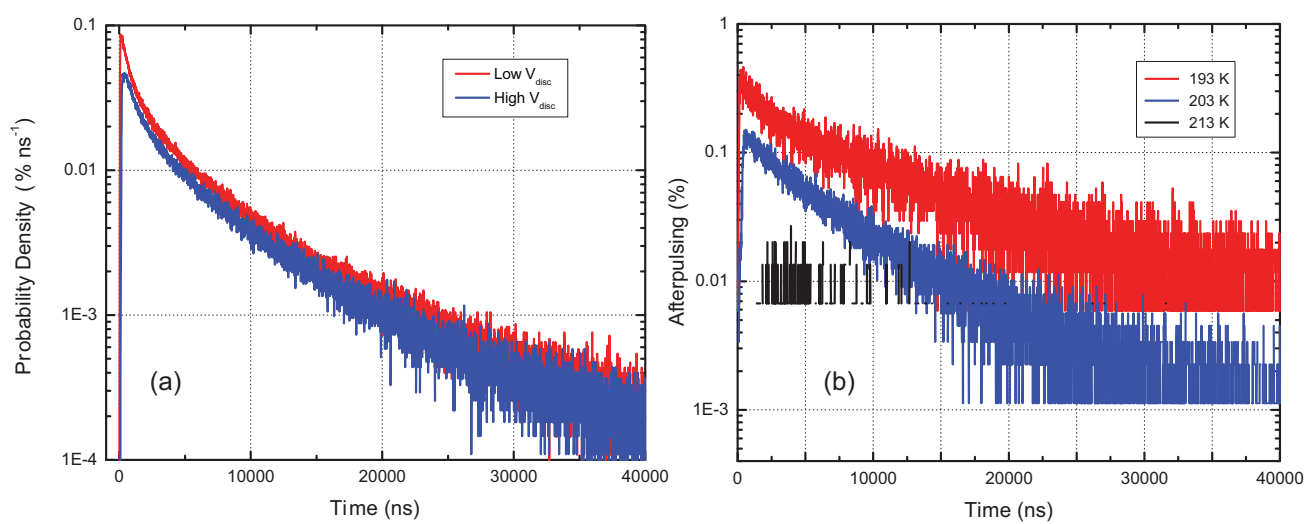

FIG. 11. (a) Afterpulsing under two threshold levels of the time tag unit input. The temperature is at 193 K. (b) Afterpulsing probability density at three distinct temperatures, while the darkcount rate was kept constant at about $100 \mathrm{CPS}$. All data are collected for $1000 \mathrm{~s}$. The threshold was set to be $0.1 \mathrm{~V}$. The reference clock rate, used to synchronize the time frame, is set to $10 \mathrm{kHz}$.
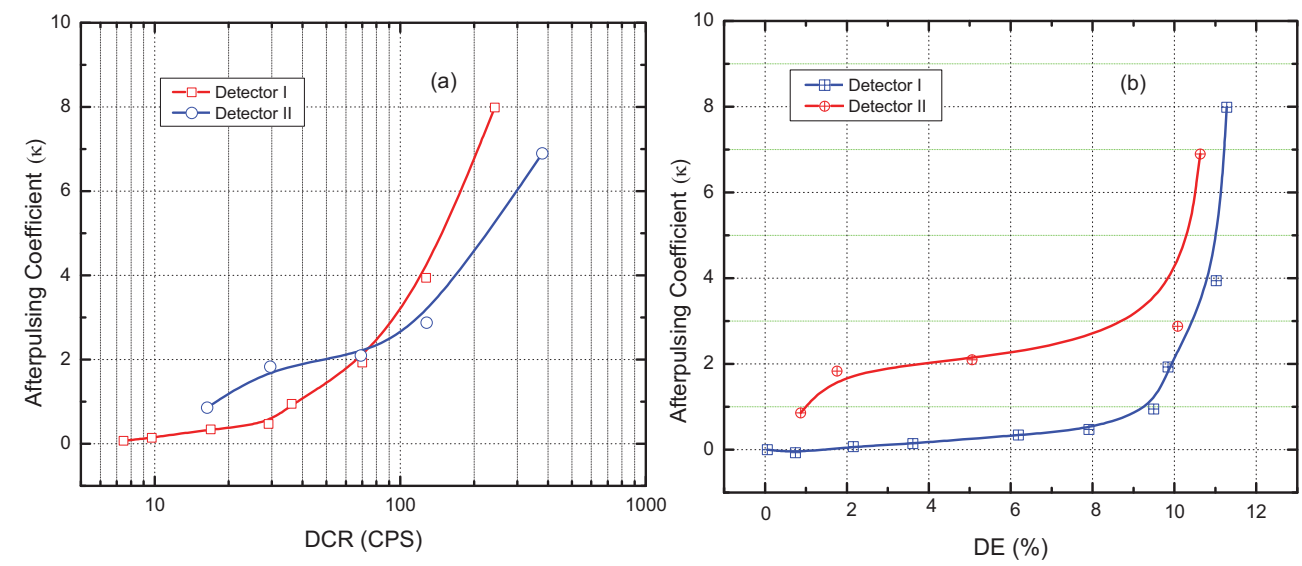

FIG. 12. Afterpulsing parameter, $\kappa$, measurement at $193 \mathrm{~K}$ by WCP characterization setup for both two NFADs. (a) The afterpulsing coefficient as a function of the darkcount rate; (b) The afterpulsing coefficient as a function of the detection efficiency. The wavelength of probing WCP pulses is $1550 \mathrm{~nm}$ for all measurements.

However, it is important to note that the measurements are performed at constant darkcount levels, and therefore the detector efficiency is lower for the higher temperature settings.

Another interesting approach to model afterpulsing behavior of a NFAD is by introducing afterpulsing coefficient defined as $\kappa=\frac{N_{t}-N_{c}-D}{N_{c}}$, where $N_{c}$ is the correlated count rate, $N_{t}$ is the total raw count rate, and $D$ is the darkcount rate at a given bias voltage. We vary the bias voltage to collect the raw counts and compute $\kappa$. The results are displayed in Fig. 12 
(a) and (b).

The comparison of the afterpulsing performance of two detectors indicates that Detector I has a better afterpulsing performance than that of Detector II. We attribute this to two factors: (1) Detector II has a larger active area than Detector I. Consequently, there are more defect trap sites in the active region that give rise to afterpulsing effects, by trapping carriers from one avalanche and releasing them at a later time when the detector becomes re-armed. (2) The larger area has a larger capacitance. This results in a proportionally larger number of charges generated when the capacitance is discharged with each avalanche. This increased charge flow per avalanche gives rise to more trapped carriers at defect sites, and thus the afterpulsing effect increases since more carriers are trapped and subsequently detrapped. The combination of these two effects creates a significant rise in afterpulsing events as the detector size is increased. The unwanted effects can be alleviated using active hold-off circuitry, which is widely used in gated mode detectors. However, we chose to keep the circuit as simple as possible, in order to avoid complexity of the electronics, its addition of stray capacitance, and undesirable elevate detector temperature.

Note that the coefficient $\kappa$ is obtained via the WCP scheme. As we mentioned in the previous section, due to changes in experimental conditions including surround light sources, the observed darkcount rates in the SPDC experiments were different than for the WCP scheme. To estimate $\kappa$ using darkcount rate for the SPDC scheme, one must take into account the joint detection efficiency.

\section{TIME RESOLUTION}

For quantum optics applications, the timing jitter of the detector, $\tau_{\text {jitter }}$, is another key parameter. The jitter time of the detector results from the fluctuations of its response time. Many mechanisms contribute to this variation. One source of jitter is the readout electronics. Another is intrinsic to the detector, primarily due to the stochastic build-up time of avalanche carriers within the multiplication region ${ }^{2}$ when the SPD detects a photon.

Here, we measured the jitter time using a Becker \& Hickl SPC-130 PCI card. We change the gain of the time to amplitude converter (TAC) to set the time resolution to $1.22 \mathrm{ps}$ per division. This setting is used for all of the measurements. The other key technique for measuring high precision jitter time is to carefully adjust the zero crossing (ZC) level of both 
inputs, usually set close the threshold levels.

TABLE I. Timing jitter measurement ${ }^{42}$ at $10 \mathrm{KHz}$ repetition rate of pulse laser, 0.1 photon and 1.0 photon on average per pulse. The measurements are accumulated for $1000 \mathrm{~s}$.

\begin{tabular}{c|c|c|c|c|c|c}
\hline \hline $\begin{array}{c}\text { Low Limit } \\
(\mathrm{mV})\end{array}$ & $\begin{array}{c}\text { ZC Level } \\
(\mathrm{mV})\end{array}$ & Total Count & Peak Count & $\begin{array}{c}\text { FWHW } \\
(\mathrm{ps})\end{array}$ & $\begin{array}{c}\text { Dark Count } \\
(\mathrm{CPS})\end{array}$ & $\begin{array}{c}\text { Average } \\
\text { Photon number }\end{array}$ \\
\hline-150 & -86 & 100261 & 799 & 154 & $\sim 150$ & 0.1 \\
-150 & -96 & 95620 & 1186 & 92 & $\sim 150$ & 0.1 \\
-140 & -96 & 96172 & 1163 & 69 & $\sim 200$ & 0.1 \\
-110 & -96 & 88066 & 1361 & 70 & $\sim 150$ & 0.1 \\
-100 & -96 & 88118 & 1405 & 59 & $\sim 150$ & 0.1 \\
-90 & -96 & - & - & - & - & 0.1 \\
\hline-150 & -96 & 706596 & 15780 & 31 & $\sim 150$ & 1.0 \\
\hline
\end{tabular}

The measurement setup is illustrated in Fig. 13 (a). The results are plotted in both logarithmic and linear scales by Fig. 13 (b) and (c), respectively. We took measurements for two different values of the average photon number per pulse. The low average photon number, 0.1 photon per pulse, effectively simulates the regime of single photon detection. At this level of $\mu$, the jitter time is mostly below 150 ps, which is compatible to the resolution of our time tag unit. This photon flux level simulates the case present in many quantum optics experiments, such as detecting photon pairs generated by nonlinear optical processes. We also set $\mu=1$, not only a comparable photon flux to what is used for decoy state QKD protoco $\frac{43}{4}$, but also to get an indication of the timing jitter intrinsic to the electronics. In all of the tests, we maintained the darkcount rate below 200 CPS.

The measurement settings and jitter measurement are also summarized in Table I. For $\mu=1$, the measured jitter is 31 ps. For $\mu=0.1$, the timing jitter becomes wider also due to the width of laser pulse ${ }^{\sqrt{2}}$, which is specified to be $<300$ ps. Most measured jitters are around 100 ps, depending on the setting of PC-130 card ranging, ranging from 59 ps to 154 ps. Most importantly, all of the detection events fall within a 400 ps time length, regardless of measurement settings, which by itself is a very good timing response. The typical response of SPADs, including silicon ones, is that their time distribution has a long tail on the order of a few ns (see Ref. 38) even if their FHWM-jitter is a few tens of ps. 

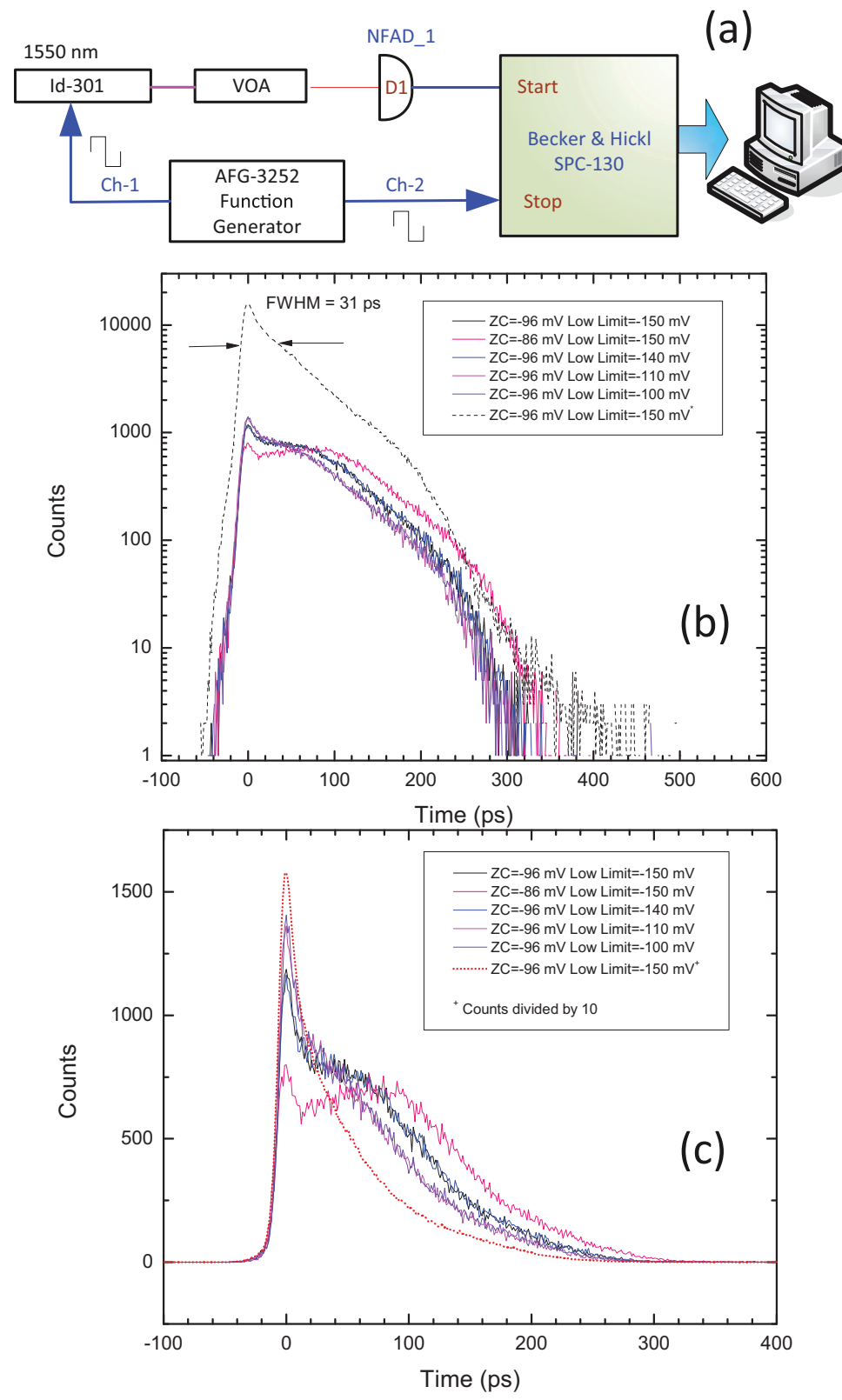

FIG. 13. Measurement of the detector jitter by Becker \& Hickl SPC-130 time-correlation card with a time resolution of 1.22 ps. (a) Experimental setup; (b) Measurements plots in logarithmic scale; (c) Normalized measurement plots in linear scale. The data are taken with 0.1 (sold lines) and 1.0 (dash line) photons per pulse for $1000 \mathrm{~s}$, with different combinations of settings to the constant-fraction discriminator (CFD), the start signal for the time measurement. The function generator (AFG 3252), which produces the stop signal, is connected to the "Synch" input of the card. The detector output is connected to CFD input after an pulse inverter transformer. The average photon number per pulse, from 0.1 to 1 , is controlled by changing the variable attenuator (VOA) settings. 
Table I also suggests a trade off between the total counts and the combination of "Low limit" and "ZC level" settings, by which we can collect higher total counts, but at the cost of longer jitter time. However, no long recovery tail is found in all of our jitter measurements. Lastly, based on these jitter measurements, the detector figure of merit ${ }^{38}$ can be calculated from the expression $H=\frac{\eta_{D}}{D \cdot \tau_{j i t t e r}}$. Our observations show that the best figure of merit is $6.3 \times 10^{7}$ for Detector 1 .

\section{SHOWING FEASIBILITY OF ENTANGLED PHOTON QKD OVER 400 KM TELECOM FIBER}

Entanglement-based QKD can achieve the longest distance when the photon pair source

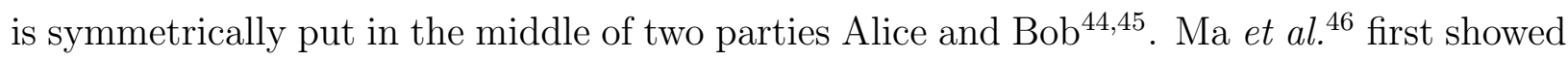
this configuration can tolerate up to $70 \mathrm{~dB}$ channel loss. Later Scheidl et al. ${ }^{47}$ performed the experiment and verified that the highest loss of their entanglement QKD achieved $71 \mathrm{~dB}$. Here, we used a similar source, i.e. a CW pumped SPDC photon pairs. The quantum channel loss is simulated using a programable optical attenuator. The experimental setup is illustrated by Fig. 14. To demonstrate the feasibility of a real QKD system, our setup has the same optical components as a standard QKD receiver, therefore we consider all potential losses from Alice, Bob and the entangled photon source.

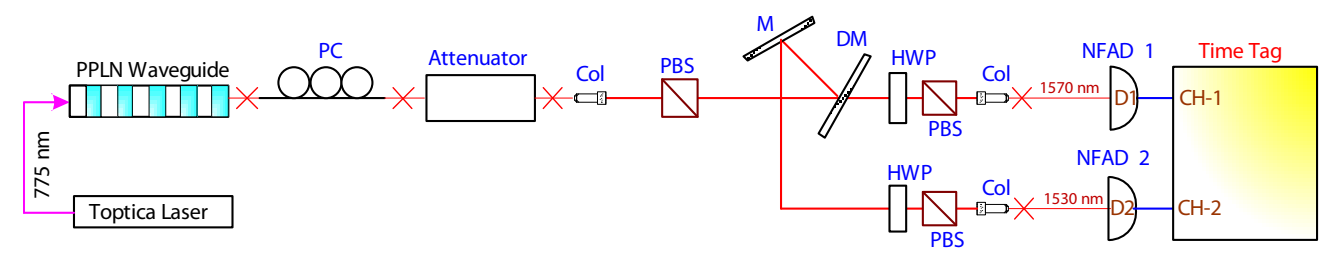

FIG. 14. Experimental setup for the investigation of High Loss QKD. Photon pairs are produced in a PPLN waveguide, and are sent through a variable attenuator. They are then split up by a dichroic mirror (DM) and detected by the NFADs. PBS: polarizing beam splitter; Attenuator: programmable optical attenuator; HWP: half wave plate; M: mirror; Col.: collimator.

We performed 68 hours of measurements to test three attenuation values set by the variable optical attenuator. We use a time tag unit to collect the counts from two NFAD detectors. The time resolution can be chosen in the postprocessing stage. The internal optical losses are characterized for two wavelengths, averaging $8.22 \mathrm{~dB}$ per arm. The total 

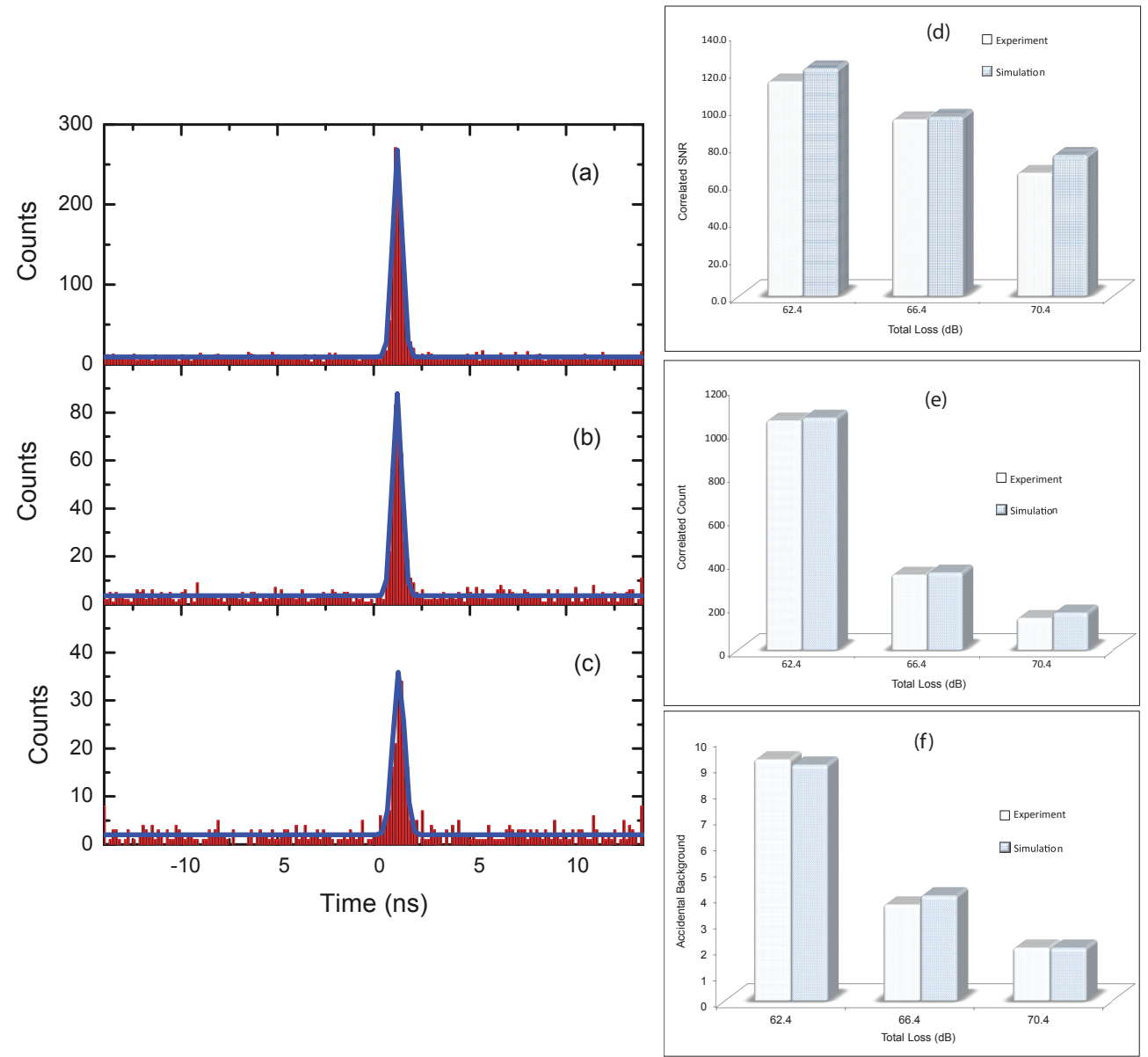

FIG. 15. The accumulated counting results collected by a 16-channel time tag unit with a time resolution of $156 \mathrm{ps}$, at three levels of attenuation settings, for (a) $62.4 \mathrm{~dB}$ and 24 hours collection time; (b) $66.4 \mathrm{~dB}$ and 20 hours collection time; (c) $70.4 \mathrm{~dB}$ and 24 hours collection time. Solid blue lines are Gaussian distribution fittings, with each of them having $\sigma=0.25 \mathrm{~ns}$, which is dominated by the timing resolution of the time tag unit. (d)-(f) are the experimental results (left) in comparison with the simulation results (right). In each of them, all above three levels of channel loss are taken into account. (d) accidentals-SNR: the ratio between the correlated and accidental counts; (e) the total correlated counts; (f) the total accidental background counts.

loss is determined to be $62.4 \mathrm{~dB}, 66.4 \mathrm{~dB}$ and $70.4 \mathrm{~dB}$ by separate measurements. The darkcount rate for both detectors is set to be below 100 CPS. Fig. 15 displays the summary of our experimental results in (a)-(c), as well as measurements in comparison with to the simulated results in (d)-(f). In the simulation, the detector efficiencies are chosen to be $\eta_{D 1}=8 \%$ at $1570 \mathrm{~nm}$ wavelength and $\eta_{D 2}=6 \%$ at $1530 \mathrm{~nm}$ wavelength. 
The analysis of the experimental data and simulation of secret key rate is based on the framework of Ref. 47. However, we only focus on the accidentals visibility by looking at the ratio between the correlated and accidental counts. We also assume our source is an SPDC source with its characteristic emission statistics, operating at the average photon pair rate $N$, is very low (4.5 MHz in our experimental measurements). $\eta_{A}$ and $\eta_{B}$ are the channel total efficiencies, each of them contains four contributions: (1) the finite detector efficiency $\left(\eta_{D 1}, \eta_{D 2}\right)$; (2) PPLN source coupling loss; (3) receiver optical loss $\left(\eta_{C A}, \eta_{C B}\right)$ and (4) link attenuation loss $\left(t_{S A}, t_{S B}\right)$, which is set by the programable attenuator. So $\eta_{A}=\eta_{C A} t_{S A} \eta_{D 1}$, and $\eta_{B}=\eta_{C B} t_{S B} \eta_{D 2}$. In general, $\eta_{A} \ll 1$ and $\eta_{B} \ll 1$ due to photon transmission losses. Based on these conditions, the lower bound of quantum bit error rate ${ }^{\sqrt{48}} Q_{B E R}$ is:

$$
Q_{B E R}=\left\{2+\frac{\eta_{A} \eta_{B}}{\mu_{P}\left[\left(1+\kappa_{A}\right) \eta_{A}+\frac{D_{A}}{N}\right]\left[\left(1+\kappa_{B}\right) \eta_{B}+\frac{D_{B}}{N}\right]}\right\}^{-1} .
$$

where the average photon pair number per time window $\mu_{P}=N W$. Here, $W$ is the time window, and $N$ is the average photon pair rate. It is limited by the detector jitter time as well as the resolution of the time tag unit. From the previous experiments, we determined that jitter time of the NFADs is shorter than $150 \mathrm{ps}$, which is shorter than the time resolution of the time tag unit 156 ps (from DotFast / UQDevices ${ }^{49}$ ). Note that the Becker \& Hickl card, which has much better time resolution, could not be used in this application because it is not suitable for the continuous time stamping of photon pair detections over the long accumulation times we require ( $\geq 20$ hours). Consequently, in all of our measurements and simulation, the smallest time window is chosen to be 156 ps. Based on this timing resolution, $N$ could be as high as $1 \mathrm{GHz}$ and still provide a 9:1 ratio for the 1-photon pair probability versus the 2 -photon pair probability. We assume $\kappa_{A}=\kappa_{B}=2$ as the afterpulsing coefficients to fit both detectors (measurements shown in Fig. 12), given the detector efficiencies in conjunction with their darkcount settings. $D_{A}$ and $D_{B}$ are the darkcount rates, and are both set to 100 CPS.

Using the QBER obtained from Eq. 3, we can estimate the secret key rate, $R$, by:

$$
R \geq \frac{1}{2}\left\{N \eta_{A} \eta_{B}\left[1-f\left(Q_{B E R}\right) H_{2}\left(Q_{B E R}\right)-H_{2}\left(Q_{B E R}\right)\right]\right\},
$$

where $H_{2}$ is the binary entropy function, $H_{2}(x)=-x \log _{2}(x)-(1-x) \log _{2}(1-x)$. The correction factor is $f\left(Q_{B E R}\right)=1.22$. 


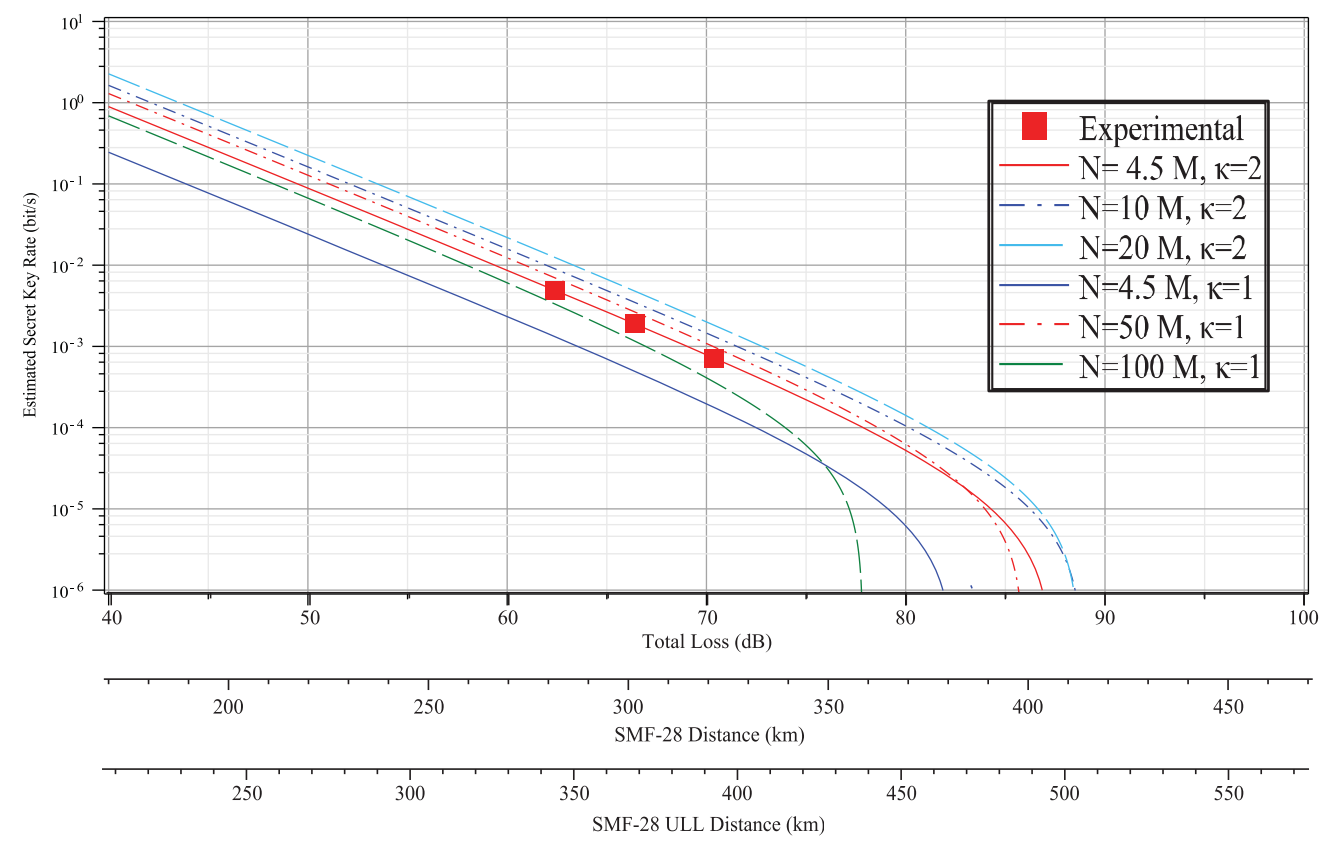

FIG. 16. The estimated secret key rate for entanglement QKD versus the total channel loss. In the numerical simulation: for the cases of $\kappa=2$, we use detector efficiency of $8 \%$ and $6 \%$, respectively; and for the cases of $\kappa=1$, two detector efficiencies are chosen to be $4 \%$ and $3 \%$, respectively. The solid squares are based on actual measurements of the signal to noise for a given loss. The lines show secret key rates calculated for different source rates and afterpulsing coefficient. Two additional x-axes show corresponding QKD distances according to the total channel loss, for standard SMF-28 single mode fiber and ultra low loss fiber, SMF-28 ULL. These results indicate that entanglement-based QKD over $400 \mathrm{~km}$ is feasible.

We experimentally simulated secret key rates for two types of telecom optical fibers: a standard telecom fiber (SMF-28) whose loss, $\alpha_{L}$, is $0.2 \mathrm{~dB}$ per $\mathrm{km}$, and an ultra low loss (ULL) telecom fiber (SMF-28-ULL) $\sqrt{50}$, whose loss is $0.164 \mathrm{~dB}$ per $\mathrm{km}$. Note that in both cases, we have introduced $6 \mathrm{~dB}$ extra loss, to account for optical losses including interconnections at Alice, Bob and central source sites. The results are showed in Fig. 16. The discrete dots in the figure are based on the experimental data for three levels of optical attenuations. The results suggest that the distance of entangled pair based QKD is feasible over $300 \mathrm{~km}$ via the standard telecom fiber, and can reach over $400 \mathrm{~km}$ in telecom ULL fiber. One caveat is that the finite key size effect has not been taken into account for both simulations. Nonetheless, given the time resolution of our detector and the possibility to 
achieve an entanglement photon source whose rate can reach up to a few hundreds of $\mathrm{MHz}$, entanglement-based QKD system over $400 \mathrm{~km}$ is possible.

Another remarkable observation is that normally a detector free of afterpulsing $(\kappa=0)$ will have its cut-off QKD distance limit proportional to the photon pair rate. This is not the case for the current NFAD design, because our detector is free running and no extra afterpulse suppressing circuit has been introduced, leading to its afterpulsing coefficient of $\kappa=2$. With this nonzero value, the QKD cut-off distance is no longer proportional to $N$. Instead the secret key rate vanishes when $N>70 \mathrm{MHz}$ approximately. However, one remedy to this situation is to decrease the current detector efficiency to $4 \%$ and $3 \%$, respectively. As a result, the afterpulsing parameter drops to less than one. Consequently, in the case of $\kappa=1$, the secret key rate does exist even when $N=100 \mathrm{MHz}$. However, as indicated in Fig. 16, its secret key rate is lower than the case when $N=20 \mathrm{MHz}$, and the efficiency of both detectors are twice as high with $\kappa=2$. Nevertheless, the regime where $\kappa=1$ will be beneficial for studying novel high rate SPDC entanglement photon pair source.

\section{CONCLUSION}

We have reported a novel design to read out single photon responses for NFAD operating at low temperatures. The system circuit diagram has been presented in detail. We carried out a SPICE model simulation to compare the conventional readout method with our approach. The simulation result agrees with the experimental measurement. Detection efficiencies of both NFADs are characterized using TCSPC measurements under different temperatures and threshold settings via WCP source. Subsequently, we have used two free running NFADs and compared them with an id-201 detector. The inferred DE of NFAD II is derived from coincidence counts of the id-201 matches the expected NFAD efficiencies obtained in WCP characterization. By scanning the average photon number in WCP, we confirmed that the NFAD acts as a bucket detector ${ }^{26}$ in the quantum optics model. We also found that the detection repetition rate can be close to $100 \mathrm{kHz}$ without DE degradation. The afterpulsing was tested by varying the threshold settings and ambient temperatures. The temporal distribution of afterpulsing events follows an exponentially decaying trend. Our measurement of the NFAD jitter time can achieve a FWHM of 31 ps. The dark noise below the rate of $10^{-7} \mathrm{~ns}^{-1}$ for the best NEP $8.1 \times 10^{-18} \mathrm{~W} \cdot \mathrm{Hz}^{-1 / 2}$. The figure of merit for 
SPDs was determined to reach $6.3 \times 10^{7}$. We also demonstrated that an entanglement-based QKD system can tolerate higher losses up to $70 \mathrm{~dB}$, which infers successful key distribution distance over $400 \mathrm{~km}$ of ULL telecom optical fiber.

We like to stress that the operating temperature for NFAD is maintained in continuously cryogenic free mode with a simple electronic circuitry. No cryogenic facility is involved; no vacuum is needed. The cooling power of our system $(10 \mathrm{~W})$ allows it to be an readily expandable platform to include higher numbers of NFADs, where the heat emitted from each detector is less than $1 \mathrm{~mW}$, even for count rates of about $1 \mathrm{MHz}$ detection pulse rate per detector. These advantages enable this NFAD system to be a good candidate in quantum optics, quantum cryptography, and SPDC entanglement source characterization applications for the telecom band.

\section{ACKNOWLEDGEMENTS}

This work is financially supported by the Natural Sciences and Engineering Research Council of Canada (NSERC), Industry Canada; Canada Foundation for Innovation (CFI), Canadian Institute for Advanced Research (CIFAR), and Early Researcher Awards (ERA)/MEDI Ontario. We are grateful for useful discussions with Dr. L. K. Shalm and Dr. H. Hübel. We wish to thank Zhenwen Wang for the technical assistance.

\section{REFERENCES}

${ }^{1}$ X. Jiang, M. A. Itzler, B. Nyman, and K. Slomkowski, in Advanced Photon Counting Techniques III, Vol. 7320 (SPIE, 2009) p. 732011 (10 pp.).

${ }^{2}$ X. Jiang, M. A. Itzler, K. O'Donnell, M. Entwistle, and K. Slomkowski, in Advanced Photon Counting Techniques V, Vol. 8033 (SPIE, 2011) p. 80330K (9 pp.).

${ }^{3}$ M. A. Itzler, X. Jiang, B. M. Onat, and K. Slomkowski, in Quantum Sensing and Nanophotonic Devices VII, Vol. 7608 (SPIE, 2010) p. 760829 (13 pp.).

${ }^{4}$ E. Knill, R. Laflamme, and G. J. Milburn, Nature 409, 46 (2001).

${ }^{5}$ C. H. Bennett and G. Brassard (1984) pp. 175-179.

${ }^{6}$ N. Gisin, G. Ribordy, W. Tittel, and H. Zbinden, Rev.Mod.Phys. 74, 145 (2002). 
${ }^{7}$ F. Stellari, F. Zappa, S. Cova, C. Porta, and J. C. Tsang, IEEE Transactions on Electron Devices 48, 2830 (2001).

${ }^{8}$ G. M. Williams and A. S. Huntington, in Spaceborne Sensors III, Vol. 6220 (SPIE, 2006) Chap. 1, pp. 622008-1.

${ }^{9}$ A. Tosi, A. Gallivanoni, F. Zappa, and S. Cova, in Advanced Photon Counting Techniques, Vol. 6372 (SPIE, 2006) pp. 63720-1.

${ }^{10}$ R. T. Thew, D. Stucki, J.-D. Gautier, H. Zbinden, and A. Rochas, Applied Physics Letters 91, $201114(2007)$.

${ }^{11}$ R. Warburton, M. Itzler, and G. Buller, Applied Physics Letters 94, 071116 (3 pp.) $(2009 / 02 / 16)$.

${ }^{12}$ J. Zhang, P. Eraerds, N. Walenta, C. Barreiro, R. Thew, and H. Zbinden (SPIE, 2010) p. $76810 Z$.

${ }^{13}$ K. Zhao, A. Zhang, Y.-H. Lo, and W. Farr, Appl. Phys. Lett. 91 (2007).

${ }^{14}$ J. Cheng, S. You, S. Rahman, and Y.-H. Lo, Optics Express 19, 15149 (2011).

${ }^{15}$ C. J. Horsfield, M. S. Rubery, J. M. Mack, C. S. Young, H. W. Herrmann, S. E. Caldwell, S. C. Evans, T. J. Sedilleo, Y. H. Kim, A. McEvoy, J. S. Milnes, J. Howorth, B. Davis, P. M. O'Gara, I. Garza, E. K. Miller, W. Stoeffl, and Z. Ali, Review of Scientific Instruments 81, $10 \mathrm{D} 318$ (2010).

${ }^{16}$ M. A. Itzler, X. Jiang, R. Ben-Michael, K. Slomkowski, M. A. Krainak, S. Wu, and X. Sun, in Enabling Photonics Technologies for Defense, Security, and Aerospace Applications III, Vol. 6572 (SPIE, 2007) pp. 65720-1.

${ }^{17}$ M. A. Albota and F. N. C. Wong, Optics Letters 29, 1449 (2004).

${ }^{18}$ A. E. Lita, A. J. Miller, and S. W. Nam, Opt. Express 16, 3032 (2008).

${ }^{19}$ G. Gol'tsman, O. Okunev, G. Chulkova, A. Lipatov, A. Semenov, K. Smirnov, B. Voronov, A. Dzardanov, C. Williams, and R. Sobolewski, Applied Physics Letters 79, 705 (2001).

${ }^{20}$ Z. Yan, A. Majedi, and S. Safavi-Naeini, Applied Superconductivity, IEEE Transactions on 17, 3789 (2007).

${ }^{21}$ V. Anant, A. J. Kerman, E. A. Dauler, J. K. W. Yang, K. M. Rosfjord, and K. K. Berggren, Opt. Express 16, 10750 (2008).

${ }^{22}$ K. Zhao, A. Zhang, Y. hwa Lo, and W. Farr, Applied Physics Letters 91, 081107 (2007).

${ }^{23}$ K. Zhao, S. You, J. Cheng, and Y. hwa Lo, Applied Physics Letters 93, 153504 (2008). 
${ }^{24}$ M. M. Hayat, M. A. Itzler, D. A. Ramirez, and G. J. Rees, in Quantum Sensing and Nanophotonic Devices VII, Vol. 7608 (SPIE, 2010) p. 76082B (8 pp.).

${ }^{25}$ X. Jiang, M. A. Itzler, B. Nyman, and K. Slomkowski, in Advanced Photon Counting Techniques III, Vol. 7320 (SPIE, 2009) p. 732011 (10 pp.).

${ }^{26}$ T. Jennewein, M. Barbieri, and A. G. White, Journal of Modern Optics 58, 276 (2011).

${ }^{27}$ J. Lundeen, A. Feito, H. Coldenstrodt-Ronge, K. Pregnell, C. Ralph, C. Silberhorn, J. Eisert, M. Plenio, and I. Walmsley, Nature Physics 5, 27 (2009).

${ }^{28}$ M. Akhlaghi, A. Majedi, and J. Lundeen, Optics Express 19, 21305 (2011).

${ }^{29}$ To determine the attenuation settings, we use a commercial power meter (OZ-300) to do the calibration. We first set a proper repletion rate $f_{\text {rep }}$ to trigger the laser, and then set a fixed attenuation value $t_{a}^{0}$ to the variable attenuator (VOA) to make sure the VOA output power $P_{0}$ is well above the noise limit of the power meter. Then the relation between average photon number $n(x)$ to the attenuator setting $x$ is $n(x)=\frac{10 \frac{P_{0}+t_{a}^{0}-x}{10} \lambda}{1000 h f_{\text {rep }}}$, where $\lambda$ is the wavelength, $h$ is the Plank constant, $c$ is the free space speed of light. Then we can solve for any arbitrary photon number $n$ with respect to attenuator setting value $x$.

${ }^{30}$ Three potential problems of the commercial id-201 detector module must be taken into account: (1) its detector temperature is monitored by the firmware; (2) we carefully adjust the delay of the arrival of the photons to make sure photon detection is within the right value; (3) the fiber connector has been carefully cleaned and inspected by the fiber scope for all of our measurements.

${ }^{31}$ B. R. Mollow and R. J. Glauber, Phys. Rev. 160, 1076 (1967).

${ }^{32}$ M. Ware and A. Migdall, Journal of Modern Optics 51, 1549 (2004).

${ }^{33}$ D. C. Burnham and D. L. Weinberg, Phys. Rev. Lett. 25, 84 (1970).

${ }^{34}$ S. V. Polyakov and A. L. Migdall, Opt. Express 15, 1390 (2007).

${ }^{35}$ P. G. Kwiat, A. M. Steinberg, R. Y. Chiao, P. H. Eberhard, and M. D. Petroff, Appl. Opt. 33, 1844 (1994).

${ }^{36}$ R. H. Hadfield, M. J. Stevens, R. P. Mirin, and S. W. Nam, J. Appl. Phys. 101, 103104 (2007).

${ }^{37}$ S. Tanzilli, W. Tittel, H. De Riedmatten, H. Zbinden, P. Baldi, M. DeMicheli, D. Ostrowsky, and N. Gisin, The European Physical Journal D - Atomic, Molecular, Optical and Plasma Physics 18, 155 (2002).

${ }^{38}$ R. H. Hadfield, Nat Photon 3, 696 (2009). 
${ }^{39}$ A. Tosi, A. D. Mora, F. Zappa, and S. Cova, Journal of Modern Optics 56, 299 (2009).

${ }^{40}$ The collection of data using constant darkcount rate about 100 CPS because it will guarantee a constant bottom line for all different measurement conditions, such as different temperatures.

${ }^{41}$ X. Jiang, M. A. Itzler, R. Ben-Michael, K. Slomkowski, M. A. Krainak, S. Wu, and X. Sun, IEEE Journal of Quantum Electronics 44, 3 (2008).

${ }^{42}$ Our setup measures the FWHM over the system level instrument response function (IRF) and combines the contributions from laser pulse jitter, electronics jitter, and NFAD detector jitter. In fact, we have only determined the upper limit of the NFAD timing jitter.

${ }^{43}$ H.-K. Lo, X. Ma, and K. Chen, Phys. Rev. Lett. 94, 230504 (2005).

${ }^{44}$ A. Scherer, R. B. Howard, B. C. Sanders, and W. Tittel, Phys. Rev. A 80, 062310 (2009).

${ }^{45}$ A. Scherer, B. C. Sanders, and W. Tittel, Opt. Express 19, 3004 (2011).

${ }^{46}$ X. Ma, C.-H. F. Fung, and H.-K. Lo, Phys. Rev. A 76, 012307 (2007).

${ }^{47}$ T. Scheidl, R. Ursin, A. Fedrizzi, S. Ramelow, X.-S. Ma, T. Herbst, R. Prevedel, L. Ratschbacher, J. Kofler, T. Jennewein, and A. Zeilinger, New Journal of Physics 11, $085002(2009)$.

${ }^{48}$ To derive our expression for the QBER, we assume $Q_{B E R}=\frac{1}{2}(1-\mathrm{Vis})$, and further assume that the entanglement visibility is $\mathrm{Vis}=\frac{\max -\min }{\max +\min }$. The coincidence counts are estimated by $N_{c}=N \eta_{A} \eta_{B}$; The accidental counts are expressed by $N_{a}=$ $\left[N \eta_{A}\left(1+\kappa_{A}\right)+D_{A}\right]\left[N \eta_{b}\left(1+\kappa_{B}\right)+D_{B}\right]$; and consequently we have $\max =N_{a}+N_{c}$, and $\min =N_{a}$. We compared the QBER given by Eq. (3) with the results in Ref. 46 to confirm that they both asymptotically agree for large losses assuming that both detectors are ideal and do not show any afterpulsing.

${ }^{49}$ Available at http://www.dotfast-consulting.at/; and http://www.uqdevices.com/.

${ }^{50}$ D. Stucki, N. Walenta, F. Vannel, R. T. Thew, N. Gisin, H. Zbinden, S. Gray, C. R. Towery, and S. Ten, New Journal of Physics 11, 075003 (2009). 